\title{
Ueber das Scammoniumharz.
}

\author{
Von H. Spirgatis. \\ (Eingegangen den 2. April 1894.)
}

Vor längerer Zeit veröffentlichte ich eine Untersuchung über (las Scammonin, ${ }^{1}$ ) das Harz von Convolvulus Scammonia Linn., in welcher ich die Identität dieses Glykosids mit dem am genauesten von W. M a y er untersuchten Jalapin, ${ }^{2}$ ) dem Harze von Convolvulus orizabensis Pell. bis auf einen so unwesentlichen Punkt feststellte, dafs diese Identität von da ab allgemein als erwiesen betrachtet wurde. ${ }^{3}$ ) Ma yer hatte nämlich bei der Spaltung des Jalapins durch Säuren neben Zucker zunächst einen fettartigen Körper erhalten, den er Jalapinol nannte, und arst beim Schmelzen desselben mit Kalihydrat Jalapinolsäure, während ich direkt bei der Spaltung die mit der Jalapinolsaure identische Scammonolsäure erhielt.

Im Jahre 1883 erschien eine Arbeit von $\mathrm{S}$ amelso $\mathrm{n}$ uber das Jalapin, ) ausgeführt, wie in derselben zu lesen ist, unter Leitung von Poleck und demselben gewidmet, welche zwar auch die Identität des Jalapins mit dem Scammonium zugab, aber doch manches von Ma $\mathrm{y} \theta \mathbf{r}^{\prime} \mathbf{s}$ und mithin auch meiner Untersuchung abweichende aufwies. Diese Arbeit war indessen mangelhaft und onthielt derartige Unrichtigkeiten und Irrtümer, dals sie unbeachtet bleiben konnte. Die freien Säuren waren nicht analysiert. Es wurde behauptet, ich hätte die Baryumverbindung der Scammonsaure durch Sättigung von Scammonsäure mit Barytwasser dargestellt, während ich sie nach derselben Methode, wie später der Autor der Dissertation, direkt aus Harz gewonnen hatte. Die über die Zusammensetzung der freien Jalapinolsäure aufgestellte Ansicht war offenbar irrig, die Analyse der jalapinolsauren Salze unrichtig, das Ma y er sche Jalapinol wurde als Aldehyd angesprochen u. w. dgl. m.

\footnotetext{
1) Annal. d. Chemie und Pharmac. CXVI 289. - Bullet. d. kgl. bayr. Akad. d. Wissenschaften 13, 106.

2) Annal. XCV 134.

3) H. Limpricht Lehrb. d. org. Chem. 1862, 624. L. Gmelin, Handbuch d. org. Chem. 1866, 1317. Wigger's Handbuch d. Pharimakognosie 1864,335 .

4) Inaugural-Dissertation, Breslau.
}

Arch. d. Pharm. CCXXXII. Bds. 4. Heft. 
Vor etwa zwei Jahren endlich veröffentlichte Poleck selbst eine Arbeit uber das Jalapin,5) welche nach seiner Angabe vornehmlich den Zweck verfolgte, die von früheren Untersuchungen abweichenden Angaben Samelson's einer Kontrolle und Revision zu unterwerfen. Diese Untersuchung hat das Verdienst, dafs sie die Irrtiumer des Schülers der Hauptsache nach berichtigt und die M a yer'schen Resultate im wesentlichen bestätigt. Auch giebt Poleck an, er habe, ähnlich wie ich bei der Spaltung des Scammonin direkt Scammonolsäure erhielt, bei der Spaltung des Jalapins sofort Jalapinolsäure erhalten und kein Jalapinol.

Einigermafsen komisch klingt es allerdings, wenn Poleck die unrichtigen Angaben seines Schülers bezüglich der Zusammensetzung der Jalapinolsäure und ihrer Salze durch die Annahme zu erklären versucht, derselbe müsse von der Aldehydnatur und $\mathrm{Zu}$ sammensetzung seines Jalapinols so "überzeugt gewesen sein, dafs er die von ihm angegebene Zusammensetzung der Jalapinolsäure und ihrer Verbindungen als eine notwendige Konsequenz der Oxydation des Jalapinols ansehen murste.

Die Wage kehrt sich nicht an Ansichten! S a mels on aber hat, wenn auch nicht die freie Jalapinolsäure, so doch Verbindungen derselben analysiert.

Uebrigens würde mich diese Arbeit $\mathrm{P}$ o le ck's, ebensowenig wie diejenige von $\mathrm{Samelson}$, zu einer Aeufserung veranlafst haben, wenn Pole c k nicht behauptete, er habe das scammonsaure Baryum anders zusammengesetzt gefunden als $\mathrm{M}$ a yer und daran, obgleich or ausdrücklich hervorhebt, dafs seine Arbeit sich ausschliefslich auf das Jalapin beziehe, die Bemerkung kntipfte: "Salze mit einem andern Baryumgehalt konnten bei wiederholten Versuchen nicht erhalten werden. Es sind daher die Angaben von W. Mayer, wie jene von Spirgatis hinfullig." Poleck giebt nämlich an, in diesem Baryumsalz 26,70 Proz. Baryum gefunden zu haben, während Mayer in demselben 21,64 und ich im scammonsauren Baryum 21,69 Baryum fand.

Obwohl es unwahrscheinlich war, dafs $\mathbf{M}$ a yer und ich für diese Salze dieselben, genau mit einander ubereinstimmenden und

5) Zeitschrift des Allgem. Oesterreich. Apotheker-Vereins 1892, No. $19,20,21$. 
trotzdem unrichtigen Zahlen erhalten haben sollten und obgleich ich bereits in meiner Untersuchung über das Scammonin (Seite 302) die Gründe angefuhrt habe, weshalb den Verbindungen der Scammonsäure, ebensowenig, wie denjenigen der Jalapinsäure ein besonderes Gewicht beizulegen ist, so habe ich doch, und zwar nach derselben Methode als früher und wie jetzt $\mathrm{Pol}$ e ck, nochmals diese Baryumverbindung aus Scammoniumharz, welches aus Scammoniumwurzel gewonnen war, durch Kochen mit Barytwasser und Entternung des Baryumüberschusses mittelst Kohlensäure dargestellt, dieselbe wie Poleck zwischen 105 und $11^{\circ}$ getrocknet und analysiert. Ich fand in Salz von drei verschiedenen Darstellungen 21,70; 21,73; 21,67 und 21,57 Prozent Baryum, mithin denselben Baryumgehalt als früher und annähernd gleiche Werte fand $\mathrm{Kr}$ omer ${ }^{6}$ ) für die in ähnlicher Weise dargestellte Scammonsaure-Verbindung. Auch die von Kromer für den $K$ ohlenst of $f$ und Wasserstoff orhaltenen Zahlen stehen denjenigen nahe, welche ich für das scammonsaure Baryum, $\mathrm{M}$ a y $\mathrm{\theta}$ r für das jalapinsaure Baryum erhielt. Allerdings vermag ich Kromer's Ansicht uber die betreffs dieser Verbindung aufgestellte Formel nicht zu teilen, weil dieselbe betrichtlich weniger Kohlenstoff verlangt, als seine Versuche ergaben.

$\begin{array}{lc}\text { Formel } & \text { Versuche } \\ \text { C } 40,61 & 41,52-41,93\end{array}$

Hiernach halte ich sämtliche Angaben, welche ich vor mehr als dreifsig Jahren uber die Zusammensetzung des Scammoniumharzes und seiner Derivate gemacht habe, in allen Stücken aufrecht und vermag nicht zu begreifen, wie Poleck über die Zusammensetzung einer Verbindung urteilen konnte, welche er seiner eigenen Angabe nach nie unter Händen hatte.

Schfiefslich erwähne ich noch, dafs $\mathrm{K}$ r omer auch bei der Analyse des jalapinsauren Baryums dieselben Werte gefunden hat, als $\mathrm{May} \theta \mathrm{r}^{7}$ ) und nicht die von Poleck angegebenen.

6) Pharmaceut. Zeitsohrift f. Rufsland 1892, 674.

7) Lbendaselbst 1892, 709. 